\title{
Approximating the Crossing Number of Apex Graphs
}

\author{
Markus Chimani $^{1}$, Petr Hliněný ${ }^{2, \star}$, and Petra Mutzel $^{1}$ \\ ${ }^{1}$ Faculty of CS, Dortmund University of Technology, Germany \\ \{markus.chimani, petra.mutzel\}@tu-dortmund. de \\ ${ }^{2}$ Faculty of Informatics, Masaryk University, Brno, Czech Republic \\ hlineny@fi.muni.cz
}

\begin{abstract}
We show that the crossing number of an apex graph, i.e. a graph $G$ from which only one vertex $v$ has to be removed to make it planar, can be approximated up to a factor of $\Delta(G-v) \cdot d(v) / 2$ by solving the vertex inserting problem, i.e. inserting a vertex plus incident edges into an optimally chosen planar embedding of a planar graph. Due to a recently developed polynomial algorithm for the latter problem, this establishes the first polynomial fixed-constant approximation algorithm for the crossing number problem of apex graphs with bounded degree.
\end{abstract}

Keywords: Crossing number, apex graph, vertex insertion.

\section{Edge and Vertex Insertion Problems}

We assume that the reader is familiar with the standard notation of terminology of graph theory, and especially with topological graphs, see [5]. A graph $G$ is called an apex graph if there is a vertex $v$ such that $G-v$ is planar. The crossing number $\operatorname{cr}(G)$ of a graph $G$ is the minimum number of pairwise edge crossings in a drawing of $G$ in the plane. Determining the crossing number of a given graph is an NP-complete problem, and exact crossing numbers are in general extremely difficult to compute.

A common heuristic way of finding a drawing of a graph $G$ with few crossings starts with a planar subgraph of $G$, and then re-inserts the remaining edges one by one in a locally optimal way. The edge insertion problem can be solved to optimality by a lineartime algorithm [3]. A subsequent result [4] uses that algorithm to give an approximation of the crossing number of almost planar graphs (i.e. those made planar by removing one edge) up to a factor of $\Delta(G)$ (recently improved to the best possible $\Delta(G) / 2$ in [1]).

A natural generalization of the previous results is to consider the problem of inserting $a$ vertex with a specified neighbourhood into a planar embedding of a graph $G$, with the least number of crossings. Although this shows to be a much harder question than that of edge insertion, a very recent result of [2] reads:

Theorem 1 (Chimani, Gutwenger, Mutzel, and Wolf). The vertex insertion problem for a planar graph can be optimally solved in polynomial time.

\footnotetext{
${ }^{\star}$ Supported by the Institute for Theoretical Computer Science ITI, project 1M0545.
} 


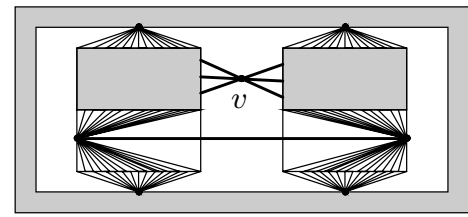

Fig. 1. An example of a vertex $v$ insertion instance requiring many crossings, eventhough the crossing number of the graph is small. The gray regions denote dense subgraphs.

\section{Crossing Number Approximation}

We can apply Theorem 1 to approximate the crossing number of apex graphs.

Theorem 2. Let $G$ be a graph and $v$ its vertex such that $G-v$ is planar, the maximum degree in $G-v$ is $\Delta$, and $v$ has degree $d$ in $G$. Then the vertex insertion problem of $v$ back into a planar embedding of $G-v$ has a solution with at most $d \cdot\lfloor\Delta / 2\rfloor \cdot \operatorname{cr}(G)$ crossings.

This new result immediately gives us a polynomial approximation algorithm for the crossing number of an apex graph $G$ up to factor $d \cdot\lfloor\Delta / 2\rfloor$. On the other hand, it is possible to construct examples for which optimal solutions to the vertex insertion problem require up to $d \cdot \Delta \cdot \operatorname{cr}(G) / 4$ crossings, cf. Fig. 1

The idea of the proof is as follows (compare to [4]): Assume $\Gamma$ is a plane embedding of the graph $G-v$ achieving optimality in the vertex $v$ insertion problem, $\Gamma^{\prime}$ is a crossing-optimal drawing of the graph $G$, and let $F$ be a minimal edge set such that $\Gamma^{\prime}-v-F$ is a plane embedding. Then $|F| \leq \operatorname{cr}(G)$ and the embedding $\Gamma^{\prime}-v-F$ can be turned into $\Gamma-F$ by a sequence of 1 - and 2-flips (Whitney flips), which allows to re-embed the edges $F$ without crossings in $G-v$. The central argument is that the number of new crossings introduced on the edges of $v$ is limited by an iteration of the following claim over all $f \in F$ :

Lemma 3. Let $H$ be an apex graph with a vertex $v$, having a drawing with $\ell$ crossings in which $H-v$ is connected and plane embedded. Let an edge $f$ connect vertices of $H-v$. If $(H-v)+f$ is planar, then there is a drawing of $H+f$ with plane embedded $(H-v)+f$ having at most $\ell+d(v) \cdot\lfloor\Delta(H-v) / 2\rfloor$ crossings.

In contrast to [4], establishing Theorem 2] using this lemma requires a careful consideration of non-biconnected graphs and the fact that the position of the newly introduced vertex $v$ is unknown and probably different between $\Gamma$ and $\Gamma^{\prime}$.

\section{References}

1. Cabello, S., Mohar, B.: Crossing and weighted crossing number of near planar graphs. In: GD 2008. LNCS, vol. 5417. Springer, Heidelberg (to appear, 2009)

2. Chimani, M., Gutwenger, C., Mutzel, P., Wolf, C.: Inserting a vertex into a planar graph. In: ACM-SIAM Symposium on Discrete Algorithms (to appear, 2009) 
3. Gutwenger, C., Mutzel, P., Weiskircher, R.: Inserting an edge into a planar graph. Algorithmica 41, 289-308 (2005)

4. Hliněný, P., Salazar, G.: On the Crossing Number of Almost Planar Graphs. In: Kaufmann, M., Wagner, D. (eds.) GD 2006. LNCS, vol. 4372, pp. 162-173. Springer, Heidelberg (2007)

5. Mohar, B., Thomassen, C.: Graphs on surfaces. Johns Hopkins Studies in the Mathematical Sciences. Johns Hopkins University Press, Baltimore MD, USA (2001) 